\title{
¿Cómo leer un estudio que va más allá de los números?: INVESTIGACI N CUALITATIVA
}

La investigación no solo contribuye al conocimiento académico sino también al desarrollo de técnicas para mejorar la práctica diaria, por lo tanto resulta de suma importancia realizar una valoración crítica de la literatura científica. Muchas veces se piensa que si un estudio se acepta para su publicación es bueno y por desgracia no siempre es así, pues la mayor parte de las investigaciones presentan diversas limitaciones y fallas.

\section{¿Qué es la investigación cualitativa?}

Preguntas como ¿cuántos padres consultan al médico general cuándo su niño tiene fiebre? ó ¿qué proporción de fumadores han intentado dejar de fumar? claramente se contestan a través de la metodología cuantitativa. Pero preguntas como ¿por qué se preocupan tanto los padres cuando sus niños tienen temperatura? ó ¿qué motiva a las personas que dejan de fumar? se resuelven con métodos cualitativos, porque para responder estas inquietudes se necesita escuchar a las personas y trabajar con el discurso. La investigación cualitativa empieza con una intención de explorar un área particular, colecciona "datos" (observaciones y/o entrevistas). En ocasiones se la usa como vehículo para generar hipótesis o identificar problemas y posteriormente aplicar una técnica positivista (método o investigación cuantitativa). No obstante, las técnicas cualitativas son útiles en sí mismas y se las utiliza con el propósito de encontrar o interpretar las respuestas de las personas o comunidades, intentando identificar el significado sociocultural que un evento dado puede tener en una comunidad. Los investigadores que usan métodos cualitativos buscan una verdad más profunda, apuntan a "las cosas en su escena natural" "intentando darle un sentido....." e "interpretando los fenómenos en términos del significado que las personas tienen de ellos" desde una perspectiva holística 1,2,3.

Aún en la actualidad hay mucha discordancia y debate sobre el criterio apropiado para la apreciación crítica de la investigación cualitativa. La evaluación de estudios cualitativos requiere considerar los méritos y las limitaciones como cualquier otro tipo de investigación. Todo informe consta de diversas dimensiones que deben tomarse en consideración al realizar una evaluación crítica. Pese a la clara diferencia con la investigación cuantitativa en general y en cuanto al contenido, los distintos tipos de informes son similares. Las diferencias principales radican en la cantidad de detalles que incluyen unos y otros y el énfasis que se concede a cada una de sus partes 4,5 .

Para juzgar la utilidad y el valor de un estudio es fundamental evaluar si la pregunta que generó el estudio está claramente formulada. Si el objetivo de la investigación es explorar, inter pretar u obtener un entendimiento más profundo de un problema clínico en particular (por ej.: creencias sobre la epilepsia y las razones percibidas por los pacientes para no tomar la medicación), los métodos cualitativos son los más apropiados para usar. Una vez analizada la pregunta que dio origen al estudio pasamos a ver si los participantes seleccionados son los más adecuados para responder a dicha pregunta 6 .En investigación cualitativa no hay un tamaño muestral definido porque se trabaja con el discurso de la gente o bien con observaciones que pueden ser uno/a ó varios/as.Se considera que tanto las entrevistas u observaciones son suficientes cuando el discurso o los hechos observados se repiten en el tiempo, es decir, cuando ya no se obtiene nada nuevo del análisis del discurso (punto de saturación) 7 .
Hay 6 preguntas básicas que como lector de trabajos científicos se deben tener presentes: 8

1) ¿Incluye el informe suficientes detalles para permitir una revisión crítica del objetivo, marco conceptual, diseño, métodos, manejos de implicancias éticas e interpretación del estudio?.

2) ¿El título del informe refleja adecuadamente la población en estudio?.

3) ¿El informe está bien ordenado o la presentación es confusa?.

4) ¿Hay un ordenamiento lógico en la presentación de ideas?.

5) ¿Las transiciones son graduales, se manifiesta continuidad de pensamiento y expresión?.

6) ¿La información es suficientemente concisa o se incluyó gran número de detalles improcedentes?.

A continuación cada una de las preguntas se desglosan respetando cada uno de los aspectos que componen un estudio:

\section{EVALUACIÓN DEL DISEÑO}

a) Dada la naturaleza del problema de investigación ¿el diseño fue el más adecuado?.

b) ¿Se describió con detalle la intervención?.

c) ¿EI $N^{\circ}$ de datos recabado u observaciones registradas fue el apropiado?.

d) ¿Se identificó y describió la población blanco o accesible?. ¿Se especificaron los criterios de elegibilidad?

e) ¿Qué procedimientos se aplicaron para controlar los factores externos (situacionales)?.¿Fueron pertinentes y adecuados?

f) ¿En que medida afectó el diseño la validez interna del estudio?.

g) ¿Cuáles son las principales limitaciones del diseño que se empleó?.¿Las reconoció el investigador?.

\section{EVALUACIÓN DE LAS TÉCNICAS DE RECOLECCIÓN DE DATOS}

a) ¿Quién recabó los datos?. ¿Fueron adecuados los recolectores o hubo algún factor (como su papel profesional, su posible relación con los sujetos, etc.) que influyera negativamente en la recolección de datos de alta calidad y sin sesgo?. 
b) ¿Cuál fue el adiestramiento que recibieron los recolectores?. ¿Se tomó alguna medida para incrementar su capacidad de estimular o generar datos de buena calidad?

c) ¿Dónde y en que circunstancia se recolectaron los datos?. ¿La presencia de otros ocasionó distorsiones?.

d) ¿Qué riesgos corrieron los sujetos al proporcionar datos para la investigación?.

e) ¿El proceso de recolección de información ocasionó algún problema a los sujetos (tiempo, tensión emocional, etc.). ¿Cómo afectó esto la calidad de los datos?.

\section{EVALUACIÓN DE LA CALIDAD DE LOS DATOS}

a) ¿Se consigan en el informe los procedimientos para incrementar o evaluar la integridad de los datos?.

b) ¿Qué técnicas se utilizaron para incrementar y evaluar la calidad de los datos?. ¿Procedimientos de triangulación, verificación por parte de informantes, supervisión externa?

c) En base a los procedimientos utilizados ¿qué confiabilidad tienen los resultados?

\section{EVALUACIÓN DEL ANÁLISIS DE LOS DATOS}

No hay ninguna regla dura y rápida sobre exactamente que detalles deben ser incluidos en esta sección del estudio. Uno simplemente debe preguntarse iincluye el estudio suficiente información acerca de los métodos usados? y ¿es este el método más adecuado para responder la pregunta de investigación?.

La sección de análisis de datos es donde el sentido puede distinguirse claramente de la cosa sin sentido. Luego de obtener un buen número de transcripciones de entrevistas o notas de campo, el investigador cualitativo genuino apenas habrá empezado.

El investigador debe encontrar una manera sistemática de analizar su o sus datos y en particular debe buscar los ejemplos de casos que parecen contradecir o desafiar las teorías derivadas de la mayoría. Una manera de hacer esto es a través de una lista de categorías, esto es "cortar y pegar" cada segmento de datos transcritos en uno o más de las categorías predeterminadas por el investigador 9 . Este proceso de codificación puede realizarse a mano o bien a través de un software (por ej.: NUDIST;THE ETHNOGRAPH; LIPSQUAL) diseñado especialmente para trabajar con este tipo de datos. Estos programas permiten almacenar, separar codificar y recuperar los datos cualitativos.

a) ¿Fue adecuada la utilización de análisis cualitativo o hubiera sido preferible utilizar un método cuantitativo?. b) ¿Cuáles fueron las fuentes de datos (por ej. entrevistas no estructuradas, entrevistas semiestructuradas, grupos focales, observaciones, etc.). ¿Fueron suficientes para proporcionar la información requerida y captar todas las variaciones posibles con respecto al fenómeno investigado?

c) ¿Se utilizaron categorías de codificación para ordenar los datos descritos?. ¿Se presentaron ejemplos de datos correspondientes a cada categoría?.¿El informe describe las reglas que se emplearon para asignar los datos a las diferentes categorías?.¿Fueron éstas lógicas y completas o se superponen y son redundantes?.

d) ¿Se describió con claridad el proceso de razonamiento con el cual se llevó a cabo el análisis temático?. ¿Cuáles son los temas que surgieron?. ¿Captan los temas el significado de los relatos, es decir, se interpretaron y conceptualizaron los temas de manera adecuada?.

e) ¿Qué método utilizó el investigador para validar los resultados?. ¿Utilizó procedimientos cuasiestadísticos? ¿Dos o más investigadores codificaron y analizaron los datos en forma independiente?.

f) ¿Se continúo la recolección de los datos hasta que se llegó a un punto de saturación?.

g) ¿Se presentan los datos de manera que el lector pueda verificar las conclusiones teóricas del estudios? ¿Se utilizó un marco conceptual para explicar los procesos más importantes?.

h) ¿Se describió de manera adecuada el contexto del fenómeno que se investigó?.

i) ¿Las relaciones entre los conceptos se describen con claridad?. ¿Tales relaciones son lógicas y reflejan con precisión los datos?

j) ¿La inclusión de datos cuantitativos mejora la calidad de la investigación?.

\section{EVALUACIÓN DE LOS RESULTADOS, IMPLICANCIAS Y RE- COMENDACIONES}

¿Son los resultados creíbles y son ellos clínicamente importantes?. En este tipo de investigación no es posible evaluar los resultados cualitativos a través de la precisión y exactitud de los métodos cuantitativos tradicionales a los que el lector puede estar más habituado, como medidas de efecto, intervalos de confianza y número necesario de pacientes a tratar (NNT). Un aspecto importante de la sección de los resultados es verificar si los autores citan datos reales. Para responder a la pregunta ¿la interpretación es congruente con los hallazgos?, sería infinitamente más creíble si se reprodujeran literalmente una o más entrevistas a modo de ilustración y confiabilidad 10.

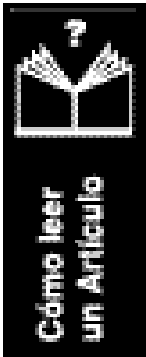

a) ¿La interpretación concede la debida consideración a las limitaciones de los métodos de investigación?. 
b) ¿Hay evidencia de sesgos?.

c) ¿Resultan congruentes las implicancias discutidas con las limitaciones del estudio?

d) ¿Se hicieron generalizaciones injustificadas con respecto a la muestra utilizada?

e) ¿Se hicieron recomendaciones acerca de la forma en que se puede mejorar el método de estudio en futuras investigaciones?.

f) ¿Se hicieron recomendaciones sobre actividades prácticas específicas en base a los resultados del estudio?
Recordar que "el tipo de estudio es el esquema general o marco estratégico que le da unidad, coherencia, secuencia y sentido prác tico a todas las actividades que se emprenden para buscar respuesta al problema y objetivos planteados". Cuando tenemos que evaluar la utilidad y la validez de un estudio cualitativo, es fundamental evaluar en forma sistemática y lograr responder adecuadamente los distintos aspectos del informe que aquí se detallaron 11.

En el área de la salud, los estudios cualitativos brindan una gran riqueza de información sobre aspectos tradicionalmente ignorados por la investigación cuantitativa y que son absolutamente necesarios para mejorar nuestra calidad de atención.Implican el desarrollo de una estrategia que permite ahondar en el conocimiento de las actitudes, creencias y preferencias que las personas pueden tener sobre una situación particular, sea esta una enfermedad, una etapa de la vida o simplemente la percepción que tienen nuestros pacientes de su propia realidad.

Dra. Nanci Giraudo [ Unidad de Medicina Familiar y Preventiva.Hospital Italiano de Buenos Aires ]

\section{Bibliografía}

1.Black N.Why we need qualitative research.J Epidemiol Community Health 1994;48:425-6.[Medline].

2.Britten N.Fisher B.Qualitative research an general practice. British Journal of General Practice 1993:43:270-271

3.Mays N, Pope C, eds.Qualitative research in health care.London:BMJ Publishing Group, 1996.

4.Krefting L.Rigor in qualitative research:the assessment of trustworthiness.Am J Occup Ther 1991;45:214-221.

5.Britten N, Jones R, Murphy E, Stacy R.Qualitative research methods in general practice and primary care.Fam Pract 1995;12:104-14.

6.Crabtree B.F., Miller W.L.1992.Research Methods for Primary Care.Vol 3."Doing Qualitative Research".

7.Patton MQ.1990."Designing Qualitative Studies", Purposeful Sampling.Cap.5, pp.169-186, en Qualitative Evaluation and Research Methods.

8. Abell P. Methodological achievements in sociology over the past few decades with specific reference to the interplay of qualitative and quantitative methods. In:Bryant C, Becker $\mathrm{H}$, eds. What has sociology achieved? London:Macmillan, 1990.

9.Patton MQ.1990."Analysis, Interpretation and Reporting".Cap.3, pp.369-506, en Qualitative Evaluation and Research Methods.

10.Taylor SJ.Y Bogdan R.1996."Introducción.Ir hacia la gente".Cap.1 en Introducción a los métodos cualitativos de investigación.Paidós, Barcelona, pp.15-27.

11.Denkin NK, Lincoln YS, eds. Handbook of qualitative research.London:Sage, 1994.

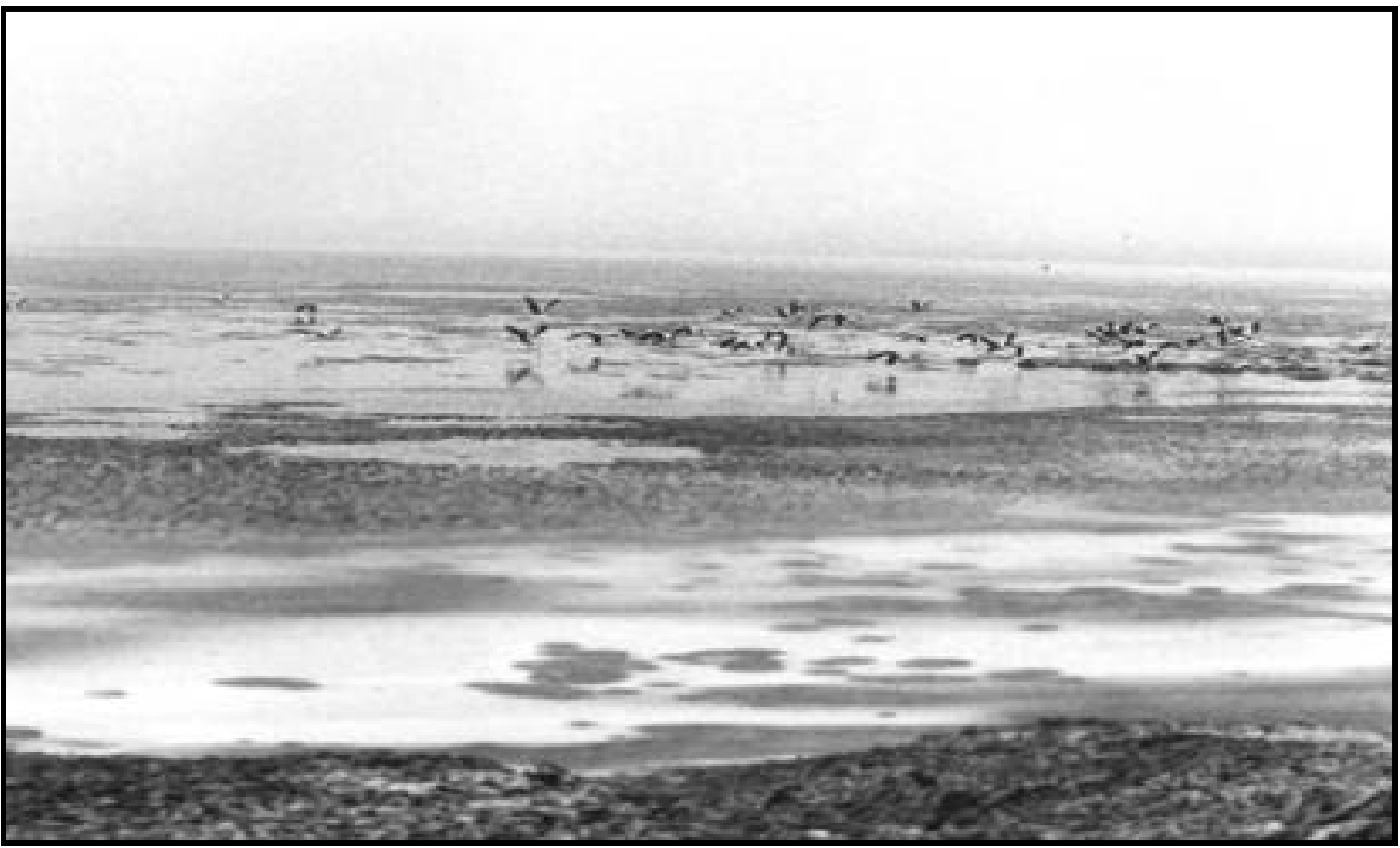

